\title{
Ares V Launch Capability Enables Future Space Telescopes
}

\author{
H. Philip Stahl \\ NASA Marshall Space Flight Center, Huntsville, AL 35812
}

\begin{abstract}
NASA's Ares V cargo launch vehicle offers the potential to completely change the paradigm of future space science mission architectures. A major finding of the NASA Advanced Telescope and Observatory Capability Roadmap Study was that current launch vehicle mass and volume constraints severely limit future space science missions. And thus, that significant technology development is required to package increasingly larger collecting apertures into existing launch shrouds. The Ares V greatly relaxes these constraints. For example, while a Delta IV has the ability to launch approximate a 4.5 meter diameter payload with a mass of $13,000 \mathrm{~kg}$ to L2, the Ares $\mathrm{V}$ is projected to have the ability to launch an 8 to 12 meter diameter payload with a mass of $60,000 \mathrm{~kg}$ to L2 and 130,000 kg to Low Earth Orbit. This paper summarizes the Ares V payload launch capability and introduces how it might enable new classes of future space telescopes such as 6 to 8 meter class monolithic primary mirror observatories, 15 meter class segmented telescopes, 6 to 8 meter class $\mathrm{x}$-ray telescopes or high-energy particle calorimeters.
\end{abstract}

Keywords: Ares V Launch Vehicle, Large Space Telescopes, Launch Capability, Astronomy

\section{INTRODUCTION}

A major finding of the NASA Advanced Telescope and Observatory Capability Roadmap Study (ATO CRM) was that current launch vehicle mass and volume constraints severely limit future space science missions' architectures, technical performance and risk. (1) And, that significant technology development is required to package increasingly larger collecting apertures into existing launch shrouds.

To date, these challenges have been overcome by fabricating systems with lower areal densities and complex packaging configurations. For example, consider the Hubble Space Telescope and the James Webb Space Telescope (JWST). Hubble had a single monolithic 2.4 meter diameter primary mirror. JWST is a 6.5 meter diameter telescope with 25 square meters of collecting aperture whose primary mirror consists of 18 separate mirror segments. Hubble's primary mirror assembly weighed slightly over $1860 \mathrm{~kg}$ for an areal density of $410 \mathrm{~kg} / \mathrm{m} 2$ and its primary mirror weighted approximately $830 \mathrm{~kg}$ for an areal density of 180 $\mathrm{kg} / \mathrm{m} 2$. By comparison, the entire JWST Optical Telescope Assembly is required to weigh less than $1300 \mathrm{~kg}$ for an areal density of $50 \mathrm{~kg} / \mathrm{m} 2$ and the JWST Primary Mirror Assembly has an areal density of approximately $30 \mathrm{~kg} / \mathrm{m} 2$. Hubble fit inside the Shuttle payload bay. But, for JWST to fit inside the $4.57 \mathrm{~m}$ diameter Ariane 5 shroud, its primary mirror is folded in a 'dropleaf' configuration (Figure 1).

As identified by the NASA ATO CRM study, similar challenges exist for future space science missions. For example, the terrestrial planet finder coronagraph (TPF-C) program is considering an elliptical asymmetric monolithic aperture. The Constellation-X program wants a 1.6 meter diameter $\mathrm{x}$-ray telescope with a mass of $2800 \mathrm{~kg}$. To achieve this, individual mirror segments must have an areal density of less than $3 \mathrm{~kg} / \mathrm{m} 2$. And, how does one package a 15 meter class segmented mirror into a 5 meter launch shroud? Furthermore, how does one minimize the potential cost, schedule and implementation risk of these increasing complex solutions?

While significant technical challenges have been met and overcome to package the 6.5 meter JWST into a 5 meter class launch vehicle, an entirely new approach is required for the desiredlarger aperture systems of the future. These can be achieved either via developing even lower areal density mirrors or more sophisticated packaging and deployment schemes or on-orbit assembly or synthetic aperture formation flying or a larger launch vehicle.

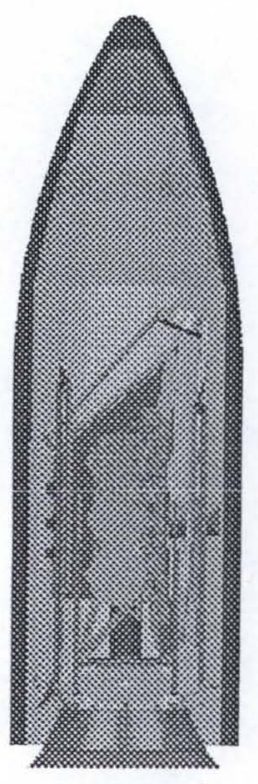

Figure 1 JWST stowed in Ariane 


\section{ARES-V LAUNCH CAPABILITY}

The planned NASA Ares V cargo launch vehicle (Figure 2) completely changes the paradigm of future space science mission concepts. It has the potential to revolutionize space astronomy by being able to place into orbit far more volume and mass than any existing system (Table 1). For example, using a lunar phasing maneuver, a Delta IV can place a 13,000 kg payload with its 5meter fairing into a transfer trajectory to the Sun-Earth L2 orbit, while the Ares V can place a $60,000 \mathrm{~kg}$ payload into the same transfer trajectory with its 8.4-meter fairing. $(2,3)$

Table 1 Launch Capabilities of an Ares V vs a Delta IV

\begin{tabular}{|l|l|l|}
\hline Launch Vehicle & Delta IV & Ares V \\
\hline Shroud Diameter & 5 meters & 8.4 meter \\
\hline Mass to Low Earth Orbit & $23,000 \mathrm{~kg}$ & $130,000 \mathrm{~kg}$ \\
\hline Mass to Earth/Sun L2 Transfer Trajectory & $13,000 \mathrm{~kg}$ & $60,000 \mathrm{~kg}$ \\
\hline
\end{tabular}

The baseline Ares V 8.4 meter shroud has a 7.5 meter dynamic inner envelope diameter and an 18 meter envelope height. (Figure 3) Alternative shrouds being considered include one with a 12 meter outer diameter, 10.3 meter payload diameter and 21 meter total height.

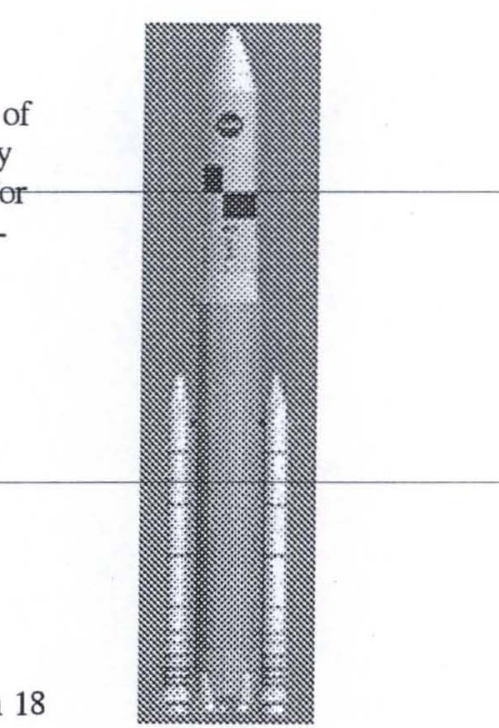

Figure 2 Baseline CaLV 8.4 m Shroud

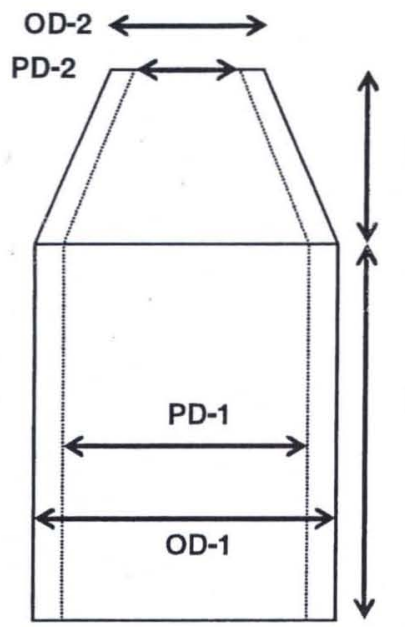

\section{Shroud Outer Diameter}

H-2

$O D=$ outer diameter

$\mathrm{PD}=$ payload dynamic envelope

$\mathrm{H}-1$
Shroud Mass

OD-1

PD-1

$\mathrm{H}-1$

OD-2

PD-2

$\mathrm{H}-2$
$8.4 \mathrm{~m}$

$5.9 \mathrm{mT}$

$8.4 \mathrm{~m}$

$7.5 \mathrm{~m}$

$12.0 \mathrm{~m}$

$4.8 \mathrm{~m}$

$3.9 \mathrm{~m}$

$6.3 \mathrm{~m}$

Total Height $(\mathrm{H}-1+\mathrm{H}-2) \quad 18.3 \mathrm{~m}$

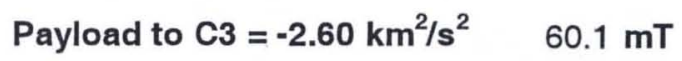

Figure 3 Ares V Baseline Shroud Dimensions and Payload Mass Capability.

Please note that these are preliminary values and may not match the latest Ares V shroud dimensions and weights.

The Ares V launch environment and potential trajectories were analyzed by the NASA MSFC Advanced Concepts Office using POST3D. (3) The maximum launch loads for the Ares V (summarized in Table 2) are similar to those for existing launch vehicles. Please note that these loads are not concurrent. They are the maximum load experienced at some time during launch. And, the lateral loads do not include wind loading or vibration.

Table 2 Maximum Launch Loads of an Ares V via POST3D Analysis

\begin{tabular}{|l|l|}
\hline Maximum Launch Load & Ares V \\
\hline Axial $(\mathrm{Z})$ & 4 g's \\
\hline Lateral $(\mathrm{Y})$ & $7 \times 10^{-6} \mathrm{~g}$ 's \\
\hline Lateral (Z) (Z is down range direction) & $6 \times 10^{-4} \mathrm{~g}$ 's \\
\hline
\end{tabular}


Two potential trajectories were analyzed: Direct Insertion and Lunar Phasing. A direct insertion trajectory has been selected for JWST and a lunar phasing trajectory was used by the Wilkinson Microwave Anisotropy Probe (WMAP). (Figure 4) Both have their advantages and disadvantages. The lunar phasing trajectory can delivery a slightly more massive payload to $\mathrm{L} 2$ because it requires less fuel. (4)

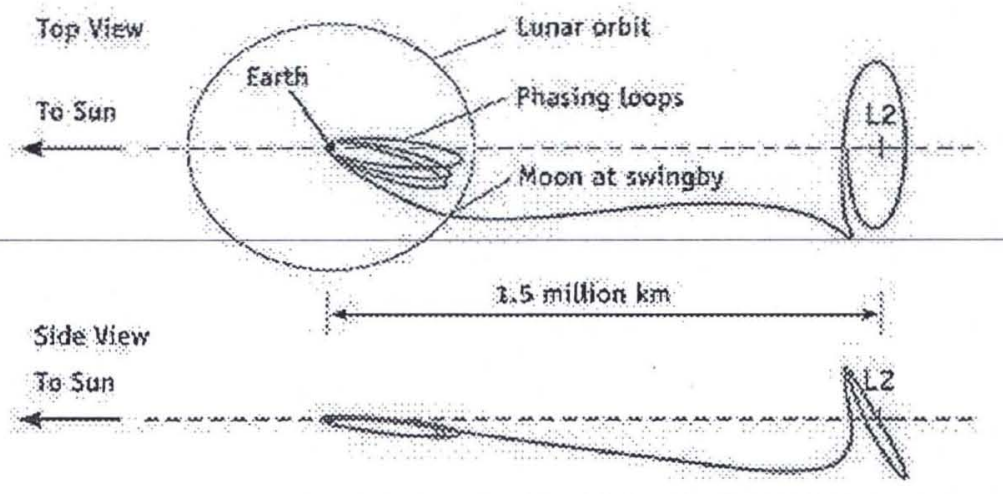

Figure 3 WMAP Transfer Trajectory from Earth to Sun-Earth L2 using Multiple Lunar Phasing Loops.

Using the trajectory and mission layout from the HTXS study report (4), the maximum mass that could be delivered to the Earth-Sun L2 Transfer Trajectory Insertion (TTI) point was analyzed for the two different trajectories using POST3D. (Table 3) The analysis assumes that the payload adapter is part of the payload mass and that the spacecraft performs all $\Delta \mathrm{V}$ maneuvers subsequent to TTI. A key parameter in Table 3 is C3 where C3 is defined as double the combined potential and kinetic energy per unit mass at TTI referenced to Earth escape velocity. A more negative value of C3 yields a larger payload capability.

Table 3 Ares V Trajectory Analysis

\begin{tabular}{|l|l|l|}
\hline Parameter & Direct Transfer & Lunar Assist with Phasing Loops \\
\hline Launch Opportunities & approx 27 days/month & approx 14 days/month \\
\hline Daily Launch Window & 5 to 10 minutes & 20 minutes \\
\hline Launch Vehicle C3 at TTI & $-0.70 \mathrm{~km} 2 / \mathrm{sec} 2$ & $-2.60 \mathrm{~km} 2 / \mathrm{sec} 2$ \\
\hline Ares V Payload to TTI Dropping Shroud & $58400 \mathrm{~kg}$ & $60100 \mathrm{~kg}$ \\
\hline Time from TTI to First Required Maneuver & 5 to 7 days & approx 8 days \\
\hline \multicolumn{2}{|l}{} \\
\hline Time from TTI to L2 & 100 days & 130 days \\
\hline Spacecraft Performs Remaining $\Delta V$ Maneuvers & $0 \mathrm{~m} / \mathrm{s}$ & $70 \mathrm{~m} / \mathrm{s}$ \\
\hline Phasing Maneuver $\Delta V$ & $5 \mathrm{~m} / \mathrm{s}$ & $5 \mathrm{~m} / \mathrm{s}$ \\
\hline Post TTI Error Correction Maneuver $\Delta V$ & $10 \mathrm{~m} / \mathrm{s}$ & $10 \mathrm{~m} / \mathrm{s}$ \\
\hline Mid-Course Maneuver $\Delta V$ & $200 \mathrm{~m} / \mathrm{s}$ & $0 \mathrm{~m} / \mathrm{s}$ \\
\hline L2 Orbit Insertion $\Delta V$ & $20 \mathrm{~m} / \mathrm{s}$ & $20 \mathrm{~m} / \mathrm{s}$ \\
\hline Station Keeping $\Delta V$ at L2 (5 years) & $235 \mathrm{~m} / \mathrm{s}$ & $105 \mathrm{~m} / \mathrm{s}$ \\
\hline Total $\Delta V$ Budget for Spacecraft Propulsion System &
\end{tabular}

The analysis assumes that the Ares V first stage has five RS-68 engines. Assisting the first stage are two 5-segment solid rocket motor boosters. The second stage is a single J2-X Block 2 engine. Transfer Trajectories and Spacecraft $\Delta \mathrm{V}$ budget were obtained from a report on the High Throughput X-ray Spectroscopy Mission, "HTXS Spacecraft and Mission Concept Study Report," HTXS Study Team Goddard Spaceflight Center, August 1997. (4)

While the cost of an Ares V cannot be known yet, it is estimated that the incremental price will be $\$ 350 \mathrm{M}$ to $\$ 500 \mathrm{M}$ per vehicle. Ares $\mathrm{V}$ vehicles will be available one per year for non-exploration payloads starting sometime after approximately 2018. 


\section{POTENTIAL SCIENCE MISSIONS}

The Ares V mass and volume payload capabilities have the potential to enable entirely new classes of mission architectures. For example, the large shroud diameter will accommodate a 6 to 8 meter class monolithic primary mirror ultraviolet/visible observatory (Figure 4) (5) or a 10 to 20 meter class segmented far-infrared telescopes. (6) Also, the large launch mass enables a 6 to 8 meter class x-ray observatory (7) or a 5 meter cube cosmic ray water calorimeter (with a mass of $130,000 \mathrm{~kg}$ ) in low Earth orbit. (8) These potential next generation very large aperture $\mathrm{x}$-ray, ultraviolet, visible, near and far-infrared space telescopes offer science performance unachievable from Earth. They provide very high angular resolution, very high sensitivity, broad spectral coverage, and high performance stability. They offer the opportunity to expand humanity's knowledge of the universe by imaging the event horizons of black holes consuming vast quantities of matter, studying dark energy and dark matter, observing galactic formation and evolution in the early universe, studying star and planetary system formation, and searching for exo-solar terrestrial planets.

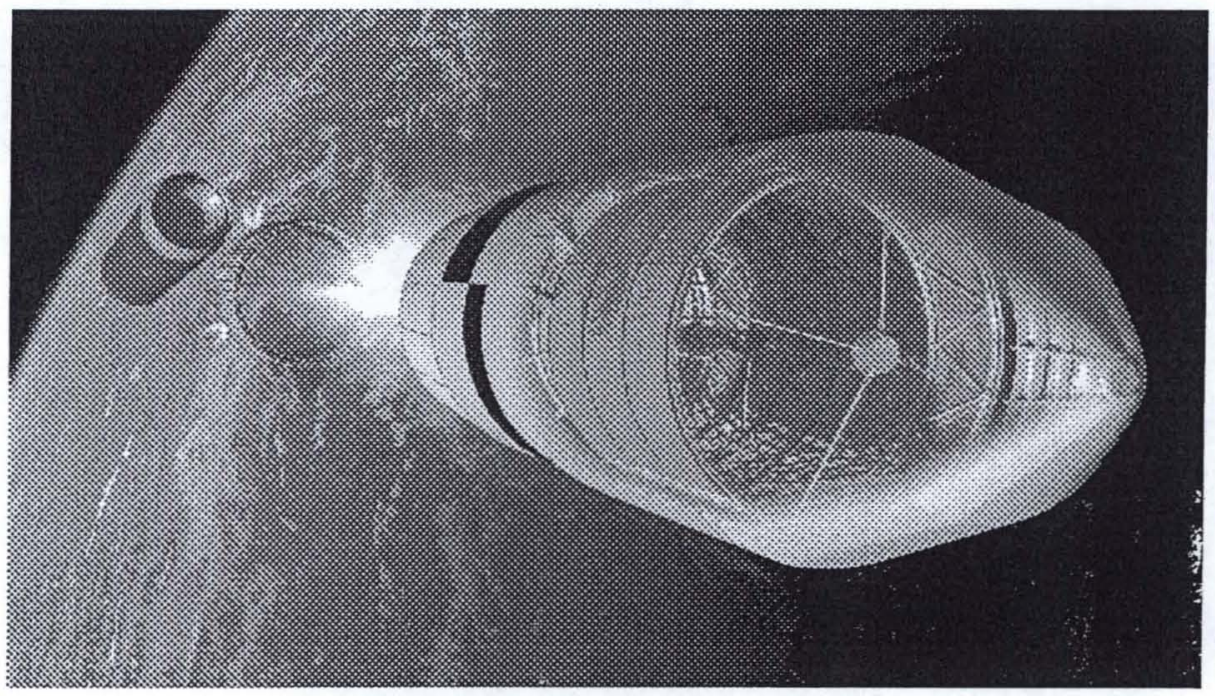

Figure 4 Ares $\mathrm{V}$ can launch 6 to 8 meter class monolithic mirror telescope.

(Image courtesy of Jack Frassanito \& Associates and Harley Thronson)

The baseline 8.4 meter Ares V launch fairing with its 7.5 meter dynamic envelop offers the ability to launch a 6 meter class monolithic primary mirror telescope. A potential 12.0 meter Ares V with a 10.3 meter dynamic shroud envelop could easily accommodate an 8 meter class monolithic mirror. A recent design study conducted at Marshall Space Flight Center has shown that it is possible to package a 6 meter class observatory into an 8.4 meter Ares V fairing (Figure 5) and have it survive launch. (3) The study telescope was an on-axis three mirror anastigmatic design with a fine steering mirror. The observatory has a 100 arc-minute $(8.4 \times 12$ arc-minutes) of diffraction limited field of view at a wavelength less than $500 \mathrm{~nm}$. The study assumed that the primary mirror would be fabricated from an existing Schott Zerodur residual VLT blank edged to 6.2 meters, $175 \mathrm{~mm}$ thick at the edge with a mass of $11,000 \mathrm{~kg}$. The entire mass budget for the observatory including primary mirror, structure, light baffle tube, instruments, space craft, avionics, etc. is less than $40,000 \mathrm{~kg}-$ a $33 \%$ mass margin on the Ares V's 60,000 kg Sun-Earth L2 launch capability. An 8 meter class observatory would have a total mass of less than $50,000 \mathrm{~kg}$ of which the primary mirror is the largest contributor. The spare VLT (Very Large Telescope) Zerodur solid thin meniscus primary mirror blank manufactured by Schott is 8.2 meter diameter, $175 \mathrm{~mm}$ thick with a mass of 23,000 kg. The LBT (Large Binocular Telescope) primary mirror blanks manufactured by the University of Arizona using their honeycomb spin cast technique are 8.4 meter diameter, $900 \mathrm{~mm}$ thick and with a mass of $16,000 \mathrm{~kg}$. Finally, the Subaru Telescope ULE thin meniscus 8.3 meter primary mirror manufactured by Corning was approximately $21,000 \mathrm{~kg}$.

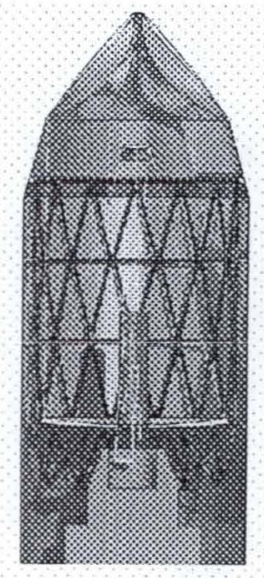

Figure 5 Monolithic 6 meter class Telescope packaged inside Ares V Dynamic Envelop. 
The use of existing ground based mirror technology for a monolithic space based telescope has two specific advantages. First, it has been demonstrated that one can actually polish an 8 meter class ground based telescope mirror to a surface figure of better than $10 \mathrm{~nm}$ rms. And second, the cost for such a mirror is approximately $\$ 20 \mathrm{M}$. The reason for both advantages is that ground based mirrors are very stiff (because they are very massive). Thus, they are much easier to fabricate than space mirrors. Historical space mirrors are very low mass and thus not very-stiff (compared to ground mirrors). They have large gravity sags and are difficult to handle, mount and fixture. And, they are difficult to fabricate to very high precision. Thus, they are expensive.

A 6-meter class panchromatic wide-field imager in space is an ideal instrument to directly address at least three key elements in NASA Origins roadmap: 1) to obtain a census of planetary systems around stars of all ages, 2) to determine chemical and physical properties of giant extrasolar planets, and 3) to determine how common are terrestrial planets. Kepler, (scheduled for launch in late 2008) will answer some of these questions. It is designed to detect planets down to earth-mass planets around F, G and $\mathrm{K}$ stars within about 200 pc. A wide-field imager on a large space telescope would complement Kepler and can be used simultaneously for planetary transit surveys as well as microlensing experiments to directly address the above questions. For example, because of its unsurpassed sensitivity, a wide-field imager should be able to detect one Earth-mass planet and 30 total planets in a single 21 day microlensing observation run. Furthermore, a 6-meter facility with a UV spectrograph would enable spectroscopic studies of the atmospheres of transiting extrasolar planets. Our understanding of the nature of extrasolar planets would be greatly increased by such a UV spectrograph, as it observes the atmospheres of short period ( 3 to 4 days) "hot Jupiters" orbiting relatively near bright stars, as they get 'boiled' away by the central star. Additionally, it would enable spectroscopy of the faint absorption lines from the 'missing baryons'. (5)

Additionally, a 6-meter class off-axis coronographic observatory using a Pupil Intensity Amplitude Apodization (PIAA) method has exceptional potential. There are 1577 F,G,K main sequence stars for which the nominal habitable zone (1 $\mathrm{AU} x$ sqrt(star luminosity)) is beyond $21 / \mathrm{D}$ on a 6-m telescope at 0.55 micron. For 365 of the 1577 IWA-accessible targets, a 6-meter observatory can realistically detect an Exo-Earth ( $>20 \%$ detection probability with $1 \mathrm{hr}$ effective Eీxposure time) and perform spestrophotometry. Of these targets, 148 are considered "eašy" (with 1 hr effective exposure tìme, $>50 \%$ detection probability in a single observation). With a 6-m PIAA telescope, $\mathrm{R}=100$ at $\mathrm{SNR}=10$ spectra of Exo-Earths can be obtained within $2 \mathrm{hrs}$ of effective exposure time around the 50 most favorable stars. (9)

And of course, large monolithic primary mirror observatories are of value for other wavelengths, for example, a farinfrared observatory. In the case of far-IR science, a 10 meter class telescope enabled by a potential 12 meter Ares V fairing is more useful than a 6 to 8 meter class mirror. A 10 meter or larger telescope, and its inherent spatial resolution, is able to better resolve very small faint objects imbedded in a bright background such as a dust cloud. It has a better confusion limit performance than a smaller aperture telescope. (6)

Even larger apertures are possible via segmentation. For example, the JWST segmented telescope deployment approach could be scaled to 10 or even 15 meters. Based on a simple diameter ratio, it is likely that the baseline Ares V 8.4 meter fairing could accommodate a 10 meter class JWST and a 12 meter class Ares V fairing could accommodate a 15 meter class JWST. Also, concepts are being explored to package up to 20 meter segmented telescopes. (6) In practice, the limiting parameter is going to be the vertical height of the Ares V fairing. Currently the baseline 8.4 meter fairing has a total height of 18 meters while the potential 12 meter fairing has a total height of 21 meters. By comparison, an Ariane V Medium fairing has 13.7 meters of height and an Ariane V Long fairing has a height of 17 meters.

One potential mission for a 10 to 15 meter segmented telescope is SAFIR (Single Aperture Far Infrared). Such a telescope would build on Spitzer and complement JWST and ALMA (which operate at shorter and longer wavelengths respectively) to study the youngest galaxies in the Universe. The high sensitivity and spatial resolution produced by a 15 meter class aperture would enable SAFIR to both detect and individually distinguish these galaxies in a manner that is much less affected by source confusion. In nearby galaxies, it could study star formation, resolve details of planetary system formation, and trace the chemical processes that lead to life. (6)

Another extremely interesting type of 6 to 8 meter class telescope is x-ray. Because $\mathrm{x}$-ray mirrors operate at grazing incidence and because they use multiple nested shells, they have very large masses per effective collecting aperture. For example, depending upon a telescope's spectral range, one square meter of effective collecting area may require 150 to 
200 square meters of mirror surface area. The current state of the art in x-ray mirror fabrication is very thin shells or segments of nickel replicated from a mandrel. The areal density for such a mirror element is approximately $3 \mathrm{~kg} / \mathrm{m} 2$. (7) Thus, 1 square meter of $\mathrm{x}$-ray collecting aperture will weight from 450 to $600 \mathrm{~kg}$. The current mission concept Constellation-X desires 6 square meters of collecting aperture with a potential mirror mass of $3000 \mathrm{~kg}$ or more. Such a mass is a serious challenge to current launch vehicles. But, as shown in the MSFC 6 meter telescope study (3), an Ares $\mathrm{V}$ can easily launch an observatory with a $15,000 \mathrm{~kg}$ primary mirror. Such a mass budget for an X-ray telescope would yield a 6.2 meter diameter mirror with 30 square meters of collecting area ( $5 \mathrm{X}$ bigger than the desired Constellation-X specification). X-ray science provided by such an observatory would highly compliment JWST by allowing the study of highly dust obscured first light object such as the first black holes, stars and galaxies. Additionally such a telescope would observe the evolution of these objects from their earliest times to the present epoch. Finally it would permit the study of matter behavior in extreme environments.

Finally, the Ares $\mathrm{V}$ enables the ability to place a large cosmic ray calorimeter in space that is capable of extending direct measurements of galactic cosmic rays beyond the $10^{15} \mathrm{eV}$ "knee" of the cosmic ray all-particle spectrum. Such a data set would allow testing of various theoretical models to explain why particles below $10^{14} \mathrm{eV}$ have different characteristics than particles above $10^{16} \mathrm{eV}$. These models include ones that predict the discovery of new cosmic ray sources, new modes of acceleration, acceleration in special classes of supernovae and propagation effects as the cause of the "knee". (8) Space is essential for this science because, due to atmospheric scattering, very high energy particles cannot be observed from Earth. But, the limiting problem has been that to detect these particles requires a very large - and thus very massive - calorimeter. The Ares V payload capacity of $130,000 \mathrm{~kg}$ to Low Earth Orbit allows the launch of a water calorimeter that is 5 meters in diameter and 6 meters in height. At 6 meters in diameter, a 3 year observation period collects sufficient particles to achieve $30 \%$ statistical accuracy at $10^{16} \mathrm{eV}$. Water has a relatively short nuclear interaction mean free path of $84 \mathrm{~cm}$ for protons. Thus, at $600 \mathrm{~cm}$ tall, proton will experience 7 interaction mean free paths. Finally, there is an added benefit. Water is one of the most valuable resources in space. It can be used for life support, energy production, propulsion and radiation protection. At the end of its scientific observing campaign, the proposed calorimeter will be available as a $>100$ metric ton reservoir of water for use in space.

\section{PROGRAMMATIC ADVANTAGES}

Almost as important as the Ares V mass and volume payload capabilities is its potential to reduce cost and performance risk and increase science return. Because all of the above described next-generation space telescopes can be built today using existing technology. It is not necessary to spending time and resources inventing new technology to package ever larger aperture telescopes into 5 meter class launch vehicles. Instead, the Ares $\mathrm{V}$ capability allows one to invest time and resources into maturing technology to reduce risk and enhance performance. Additionally, it allows one to start investing in developing technology to enable even more advanced mission concepts such as synthetic aperture observatories via formation flying or 100 meter class apertures via on-orbit assembly - missions which will also benefit from the launch capabilities of an Ares V.

\section{CONCLUSION}

NASA's planned Ares V cargo launch vehicle completely changes the paradigm of future space science mission architectures. Its ability to place $130,000 \mathrm{~kg}$ of payload into Low Earth Orbit and 60,000 kg of payload into Sun-Earth L2 inside of an 8 to 12 meter diameter fairing enables entirely new generations of space telescopes. Space telescopes enabled by an Ares V include: a 6 to 8 meter class monolithic primary mirror ultraviolet/visible observatory; a 15 to 20 meter class segmented far-infrared telescope; a 6 to 8 meter class x-ray observatory; or a 5 meter cube cosmic ray water calorimeter. These potential next generation observatories offer very high angular resolution, very high sensitivity, broad spectral coverage, and high performance stability. But of equal importance is that they do not require any new technology. Because of the Ares V's mass and volume capacities, these observatories can be built today with existing technology. Thus, allowing NASA to invest its time and resources to mature technology to reduce risk and enhance performance. And, to start investing in developing technology to enable even more advanced mission concepts such as synthetic aperture observatories via formation flying or 100 meter class apertures via on-orbit assembly. While the cost of an Ares $\mathrm{V}$ cannot be known yet, it is estimated that the incremental price will be $\$ 350 \mathrm{M}$ to $\$ 500 \mathrm{M}$. 


\section{ACKNOWLEDGEMENTS}

The author would like to thank Randall Hopkins and Alan Philips of the Marshall Space Flight Center Advaneed Concepts Office who provided information, analysis, data and valuable guidance regarding the Ares V capabilities and preliminary shroud designs. Additionally, the author wishes to recognize numerous and on-going contributions from Dan Lester of University of Texas, Marc Postman of the Space Telescope Science Institute, Brian Ramsey of Marshall Space Flight Center and Harley Thronson of Goddard Space Flight Center.

\section{REFERENCES}

1. Stahl, H. Philip, "Mirror Technology Roadmap for Optical/IR/FIR Space Telescopes", SPIE Proc.6265, (2006)

2. Stahl, H. Philip, "Ares V an Enabling Capability for Future Space Science Missions", Workshop on Science Associated with Lunar Exploration Architecture, Tempe, AZ, Feb. 28, 2007

3. “Ares V L2 Telescope Study Final Report", MSFC Advanced Concepts Office, NASA, 2007

4. "HTXS Spacecraft and Mission Concept Study Report," GSFC HTXS Study Team, NASA, 1997

5. Postman, Marc, et. al., "A Large Monolithic-Aperture Optical/UV Serviceable Space Telescope Deployed to L2 by an Ares-V Cargo Launch Vehicle", Workshop on Science Associated with Lunar Exploration Architecture, Tempe, AZ, Feb. 28,2007

6. Lester, Dan, et. al., Large Infrared Telescopes in the Exploration Era - SAFIR, SPIE Proc.6687, (2007).

7. Ramsey, Brian and Mikhail V. Gubarev, Private Communication, 2007

8. Adams, James H., et. al., Orbiting Astrophysical Spectrometer in Space (OASIS), Workshop on Science Associated with Lunar Exploration Architecture, Tempe, AZ, Feb. 28, 2007

9. Guyon, Olivier, Private Communication, 2007 


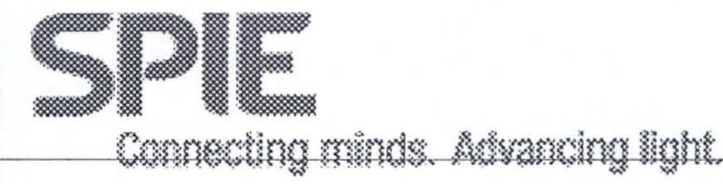

Sore to an intemationa socety abvanchn an interchisciphary approach to the science and anplication of light.
Howne

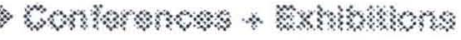

Pulalication

Courses

Memberstip

Pescources

Whack

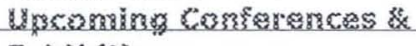

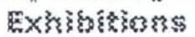

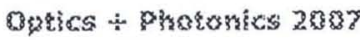

Overview

- Optical Engineening + Applications

- Naroscience + Enginerny

- Photonic Devaces + Applications

- Solar Energy + ipplications

Exibiton Overvew

Pote!, Thvel anc vente

fegistration

\section{Calls for gapers}

\section{Past. Comseremces \&}

\section{Exŷibrtions}

frformation for:

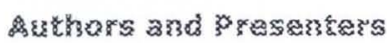

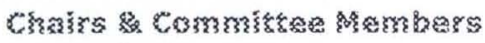

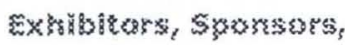

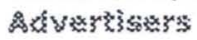

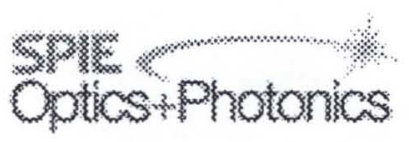

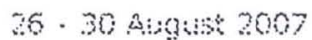

Jan biego Conventon Center

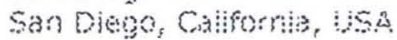

\section{Gyervinew}

A

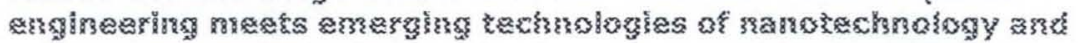

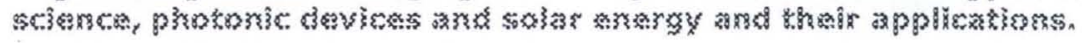

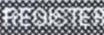

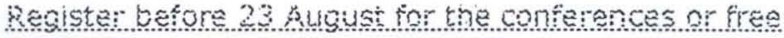
is xhibition and sove time oyjer bnsits rebistiation.

Wear the lateat reseatch from fout intormational sympustis:

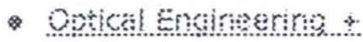
Abplications

* Aunscieno Enomerin

- Giotonic Devices - Apolications

- Solar Eneray+Aostorions

Dorit miss the premie: meating on ereming ischnigis:

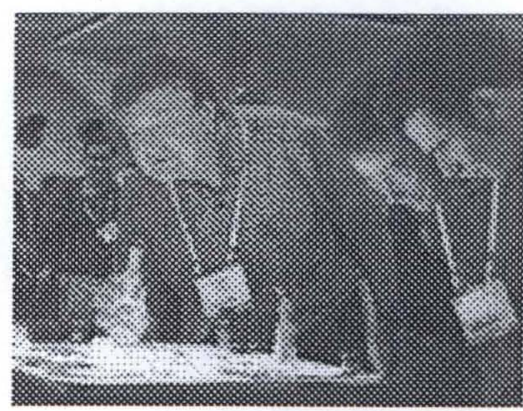

* 3,100 techrich presentations

* 70 conises

- 5,000 scentists, emomers managen and instumentaton inmovators

200 exibitots

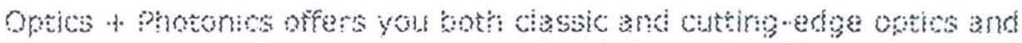

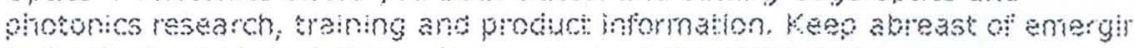

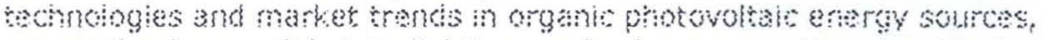
nanotechnology, solic state lighting; and solar anergy. Hear hop-lavel soeakan

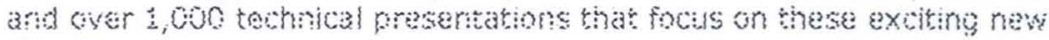
deviepments.

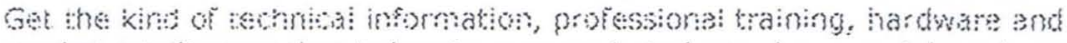
market intellgence that is key bo auccess in toudy s changing job and

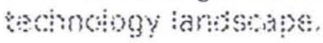


